\title{
Aquaporins and their implications on seeds: A brief review
}

\section{G. P. Chinnasamy*}

Department of Seed Science and Technology, Tamil Nadu Agricultural University, Coimbatore - 641003 (Tamil Nadu), India

\section{S. Sundareswaran}

Seed Centre, Tamil Nadu Agricultural University, Coimbatore - 641003 (Tamil Nadu), India

\section{K. S. Subramanian}

Director of Research, Tamil Nadu Agricultural University, Coimbatore - 641003 (Tamil Nadu), India

\section{K. Raja}

Department of Nano Science and Technology, Tamil Nadu Agricultural University, Coimbatore - 641003 (Tamil Nadu), India

\section{P. R. Renganayaki}

Department of Seed Science and Technology, Tamil Nadu Agricultural University, Coimbatore - 641003 (Tamil Nadu), India

\section{S. Marimuthu}

Department of Nano Science and Technology, Tamil Nadu Agricultural University, Coimbatore - 641003 (Tamil Nadu), India

*Corresponding author. Email: chinnasamy1051@gmail.com

\section{Article Info}

https://doi.org/10.31018/

jans.v13i3.2830

Received: July 8, 2021

Revised: August 22, 2021

Accepted: August 28, 2021

\section{How to Cite}

Chinnasamy, G.P. et al. (2021). Aquaporins and their implications on seeds: A brief review. Journal of Applied and Natural Science, 13(3), 970 - 980. https://doi.org/10.31018/jans.v13i3.2830

\begin{abstract}
Aquaporins (AQPs) are water channel proteins. They play a key role in maintaining water balance and homeostasis in cells under stress conditions in living organisms. AQPs are pore forming transmembrane proteins that facilitate water movement and various small neutral solutes across cellular membranes. Aquaporin expression and transport functions are modulated by various phytohormones mediated signalling in plants. Transcriptome analysis revealed the role of aquaporins in regulating hydraulic conductance in plant roots and leaves. Different AQPs found in the seed system have individual functions that are more time and tissue specific, ultimately helping in the seed imbibition process to complete seed germination. Seed specific TIP3s aquaporin helps to maintain seed longevity under expressional control of $\mathrm{ABI} 3$ during seed maturation and heat shock proteins and late embryogenic abundant proteins. Under stress circumstances, the major significance of aquaporin expression in seeds is to maintain water influx and efflux rates, as well as protein modification, post translational alterations, nutritional acquisition and allocation, subcellular trafficking and $\mathrm{CO}_{2}$ transport. The present review mainly focused on aquaporin structure, classification, role and functional activity during solute transport, reproductive organs development, plant growth development, abiotic stress response and also various roles in seeds such as seed biology, seed development and maturation, seed dormancy, seed germination and longevity.
\end{abstract}

Keywords: Aquaporins, Dormancy, Gene expression, Seed development, Seed germination

\section{INTRODUCTION}

The role of aquaporin is in regulating the fast and passive movement of water across cell membrane, which is facilitated by the presence of water channels. In addition to water, some aquaporins also transport glycerol, $\mathrm{CO}_{2}$, urea, ammonia, hydrogen peroxide, boron, silicon, arsenite, antimonite, lactic acid and metalloids. Aquaporins are localized in the plasma membrane, endo- plasmic reticulum, plastids and vacuoles. Water influx into the plant cell occurs through water channels formed by plasmalemma aquaporins (PIPs), while the delivery of excessive water to the vacuole depends on tonoplast aquaporins (TIPs) (Wang et al., 2020).

The effectiveness of water transport is determined by the amount of aquaporin in the membranes and whether the water channels are open or closed, which are primarily controlled by phosphorylation and 
dephosphorylation, respectively (Maurel et al., 2008). The water transport activity of plant aquaporin was first established in Arabidopsis (Soto et al., 2010).

In 1992, Peter Agre reported the first high-resolution images of 3D structure of an aquaporin, namely Aquaporin-I. In 2003, Peter Agre was awarded Nobel Prize for his pioneering work on the discovery of water channels.

A water molecule can pass through the small spaces, their presence between the phospholipid of the lipid bilayer. Middle part of the lipid bilayer is hydrophobic nature. The transport of water molecules will be very slow, while passingthrough the cell membrane (Knepper and Inoue, 1997) (Fig 1). In this case, aquaporins are a highly useful way to transport water molecules in plants.

\section{Structure of Aquaporin}

Aquaporin consists of six transmembrane helices with $\mathrm{N}$ and $\mathrm{C}$ termini facing the cytosol. The assembly of four aquaporin monomers forms tetrameric holoproteins. Hydrogen bonding and interactions between monomer loops keep tetramers stable. Each monomer forms a single water hole. The electrostatic interactions of proteins cause water molecules travelling through the channel to flip at the channel's core. They also have five loops (A-E) joining to the transmembrane helices as part of their structure. Two conserved loops ( $B$ and E) are extremely hydrophobic. They contain a residue that forms NPA motif (Asparagine - Proline - Alanine), which is extended into the pore from both sides of the membrane. Loop $C$ also connects to the loop $B$ and $E$.

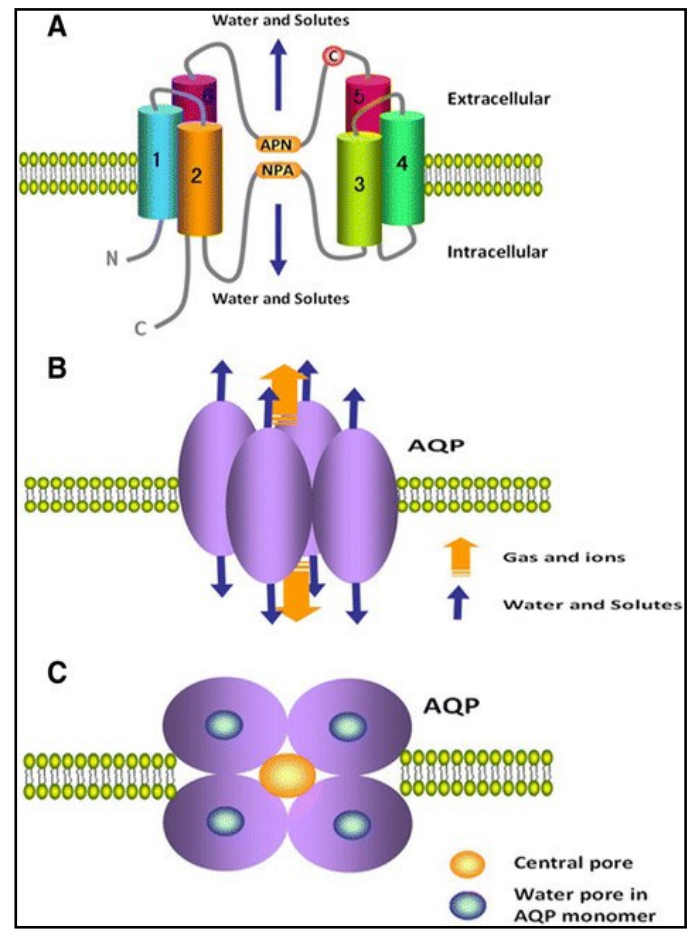

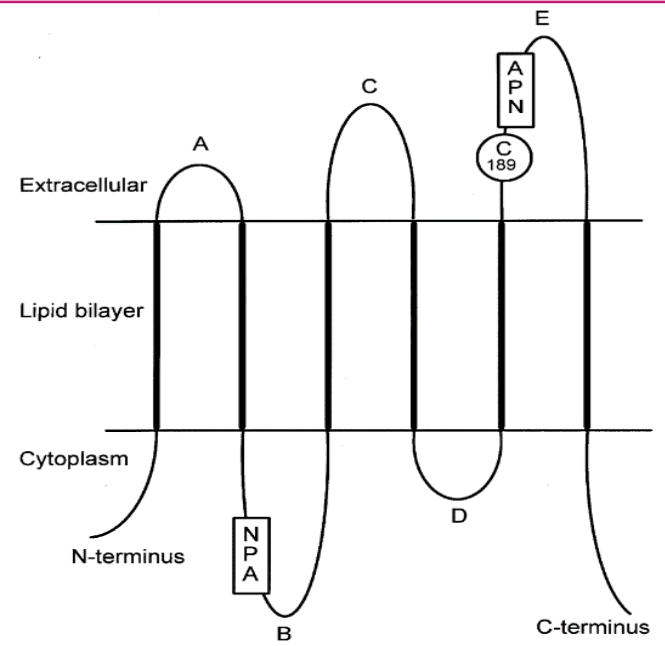

Fig. 1. General topology of aquaporin or water channel protein (Source: Knepper and Inoue, 1997).

This connection is functionally necessary for water permeability (Shapiguzov et al., 2004) (Fig. 2).

\section{Classification of major intrinsic proteins (MIPs) and Aquaporins (AQPs)}

MIPs are classified into three types based on their substrate specificity and sequence similarities: AQPs (Aquaporins), which are involved in water and ion transport; GLPs (Glycerol Facilitators), which are permeable to glycerol and neutral molecules; and GLAs (Aquaglyceroporins), which are permeable to both water and glycerol (Wang et al., 2020).

Many AQPs have different localisation, substrate specificities, transcriptional and posttranslational regulation,

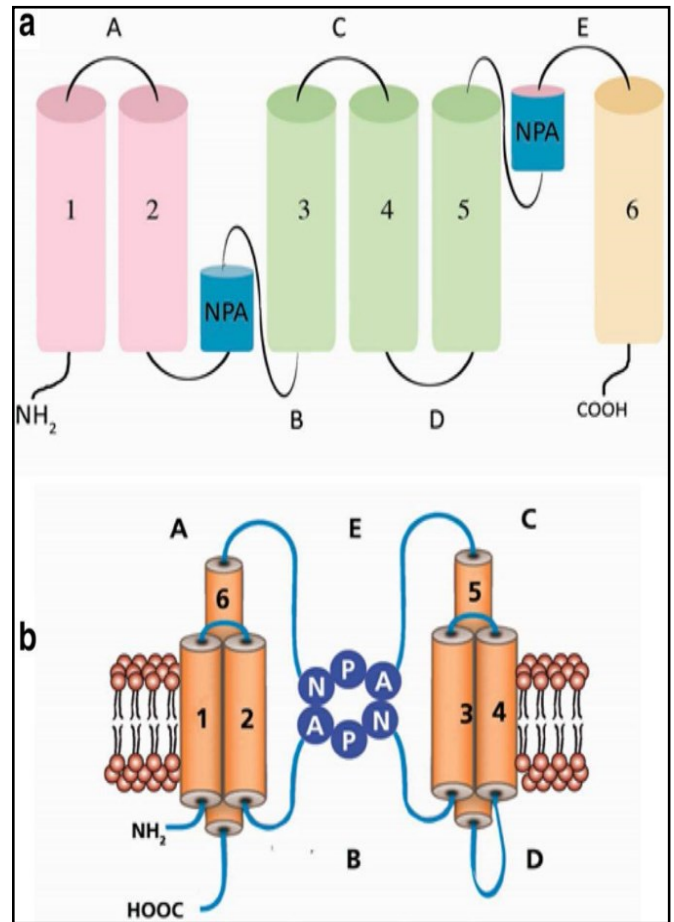

Fig. 2. Structure of aquaporin (Source: Shapiguzov et al., 2004). 
and substrate specificities (Danielson et al., 2008). AQPs are divided into five subfamilies based on membrane location and amino acid sequence: plasma membrane intrinsic proteins (PIPs), tonoplast intrinsic proteins (TIPs), NOD26-like intrinsic proteins (NIPs), small basic intrinsic proteins (SIPs) and $X$ intrinsic proteins (XIPs). PIPs and TIPs are the most common of these and they primarily mediate water transport across cells and subcellular compartments in plants. PIPs are further separated into two subgroups: PIP1 and PIP2. PIP1;1, PIP1;2, and other isoforms are found in each subgroup. TIPs are divided into TIP1, TIP2, TIP3, TIP4, and TIP5 subtypes, with isoforms within each subtype being designated as TIP1;1, TIP1;2, and so on. NIPs are also divided into NIPIs, NIPIls and NIPIIls based on their water channel pore structures (Wang et al., 2020). $\mathrm{AQP}$ isoforms are found in a variety of crop species. They have different roles in the growth and development of plants. Arabidopsis aquaporin isoform gene has predominantly expressed under stress situations among the many aquaporin isoforms (Johansson et al., 1998) (Table 1).

\section{Functions of Aquaporins}

NIPs play a similar role as water transporters and small solutes; compared to other aquaporins, NIPs have lower water permeability. SIPs are responsible for moderate water transport. Water, metalloids, and reactive oxygen species are all permeable to XIPs (Liu et al., 2013). The bulk of PIPs have been found in plasma membranes, and they are mostly found in organs with high water fluxes, such as vascular tissues, guard cells, and flowers. PIPs functions as the transporters of water, glycerol, $\mathrm{H}_{2} \mathrm{O}_{2}, \mathrm{CO}_{2}$ and urea. TIPs controls water exchange between cytosolic and vacuolar compartments, which means that they have a role in regulating cell turgor pressure. TIPs function as glycerol, urea, and ammonia transport and abiotic stress response. The main role of TIPs is the permeability of water, leaf expansion, root elongation and seed germination (Ayadi et al., 2019).

NIPs play a crucial function in water transport between bacteria and the host plant. NIPs differ from other plant MIPs in that they are found in both leguminous and non -leguminous plants' plasma and intracellular membranes. NIPs have a similar purpose as water and small solute transporters, although their water permeability is lower than that of other aquaporins. SIPs serve as a moderate water source (Wang et al., 2020).

\section{Role of AQPs in seed biology}

\section{Petal expansion and flowering}

The RhPIP2;1 aquaporin gene is primarily expressed in petal epidermal cells, according to Ma et al. (2008), and its expression is effectively connected to petal elongation in roses. Ethylene is commonly thought to be a
Table 1. Aquaporin isoforms in different crop species (Source: Johansson et al., 1998).

\begin{tabular}{ll}
\hline Crops & Aquaporin isoforms \\
\hline Maize & 31 \\
Rice & 33 \\
Sweet orange & 34 \\
Tomato & 47 \\
Soyabean & 66 \\
Banana & 50 \\
Cotton & 71 \\
Sorghum & 41 \\
Arabidopsis & 35 \\
\hline
\end{tabular}

side consequence of organ growth (Ma et al., 2008). Ethylene influences petal growth by altering the expression of RhPIP2;1, according to Dubois et al. (2018). This treatment inhibits petal cell proliferation and produces a decrease in water content in rose petals. Furthermore, ethylene suppresses RhPIP2;1 expression, and ethylene-treated flowers resemble RhPIP2; 1 silenced plants in terms of morphological properties of the petals. As a result, ethylene controls petal growth and may rely on RhPIP2;1's functions. Furthermore, the RhPIP1;1 aquaporin gene can interact with RhPIP2; 1 to increase the activity of RhPIP2;1, even though RhPIP1; 1 is incapable of carrying water on its own. Furthermore, RhPIP1;1 inhibition significantly reduces petal development (Chen et al., 2013). RhTIP1;1 has also been shown to be expressed preferentially in rose petals. Its expression is strongly linked to the blooming process and is inhibited by ethylene treatment. (Xue et al., 2009). In brassica, ginseng and arabidopsis transgenic plants overexpressing of BnPIP1, PgTIP1 and AtPIP1;2 respectively and also exhibit the phenotype of precocious flowering as compared with wild type (WT) plants. Finally, the author indicating that PIPs and TIPs have positive roles in flowering (Wang et al., 2019) (Fig. 3a).

\section{Anther dehydration and dehiscence}

NtPIP1 and NtPIP2 were discovered to be actively expressed in the anther by Bots et al. (2005). During anther development, NtPIP2 protein levels are controlled. Furthermore, RNAi-mediated downregulation of NtPIP2 resulted in a significant delay in anther dehydration and dehiscence when compared to control tobacco plants (Fig. 3b) (Table 2). Rice anther OsPIP1;1 and OsPIP4;1 are highly expressed (Table 2) (Liu et al., 2013). In Brassica, PIP1s aquaporin is also expressed in the anther (Dixit et al., 2001). PIPs are required for the dehydration and dehiscence of anthers, according to these studies (Wang et al., 2020). 


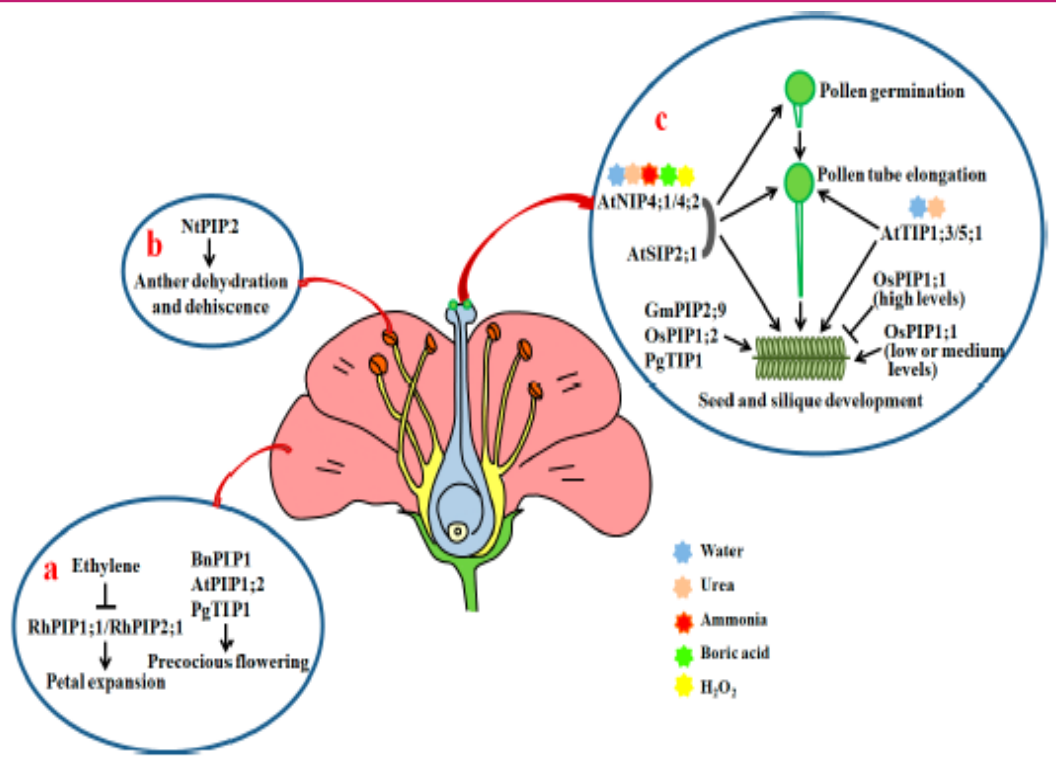

Fig. 3. (a-c). a) Role of $A Q P$ s in petal expansion and flowering; b) anther dehydration and dehiscence; c) pollen germination, pollen tube elongation and seed development (Source: Wang et al., 2020).

\section{Pollen germination and pollen tube elongation}

AtSIP2; 1 is found in the endoplasmic reticulum (ER), according to Sato and Maeshima (2019). A mutation in AtSIP2;1 results in a significant decrease in pollen germination rate when compared to wild type (WT). Furthermore, atsip2;1 pollen tubes are significantly shorter than WT pollen tubes, and most atsip2;1 pollen tubes stop elongating in the middle of the pistil (Wang et al., 2020). Seeds in the lower area of atsip2;1 siliques are likewise sparse, and atsip2;1 siliques are clearly shorter than WT siliques. Furthermore, atsip2;1 pollen has much greater transcriptional levels of a critical ER stress triggered gene binding protein 3 than WT pollen. This suggests that AtSIP2; 1 improves pollen germination and pollen tube elongation in Arabidopsis, possibly by reducing ER stress (Table 2 ).

AtTIP1;3 and AtTIP5;1 are pollen-specific proteins in Arabidopsis. AtTIP1;3 transcripts are mostly found in vegetative cells, whereas AtTIP5;1 transcripts are mostly found in pollen sperm cells (Soto et al., 2010; Wudick et al., 2014). Under nitrogen (N) deprivation conditions, the pollen tube elongation of single mutants attip $1 ; 3$ and attip5;1, as well as double mutant tip1;3/ tip5;1, is suppressed to the control. Furthermore, AtTIP1;3 and AtTIP5;1 loss of function mutations significantly increase the anomalous rate of barren siliques. As a result, pollen formation and pollen tube expansion need both AtTIP1;3 and AtTIP5;1 (Wudick et al., 2014). Because AtTIP1;3 and AtTIP5;1 operate as water and urea channels in mature pollen to remobilize nitrogen, the two AQPs may function in Arabidopsis by transporting nitrogen (Soto et al., 2008; Soto et al., 2010). AtNIP4;1 and AtNIP4;2 are also pollen-specific aquaporins, having permeability to ammonia, urea, boric acid, and $\mathrm{H}_{2} \mathrm{O}_{2}$ in addition to water. With the lowest transcription activity, AtNIP4; 1 is active in ripe pollen and pollen tubes. AtNIP4;2 is fully expressed in pollen tubes, and its expression levels rise considerably as the pollen tube grows. Pollen germination and pollen tube elongation are significantly inhibited when the expression of AtNIP4; 1 and AtNIP4;2 is reduced by RNAi (Fig. 3c) (Table 2) (Wang et al., 2019).

Overexpression of OsPIP1;1 at extremely high levels in rice reduces fertility significantly, whereas expression at low or medium levels increases seed production but has little effect on single grain weight. According to the author, OsPIP1;1 functions in a seed setting by influencing pollen germination and pollen tube development (Table 2) (Liu et al., 2020).

\section{Role of AQPs in seed development}

AQPs are mainly involved in the transportation of water and solutes during seed development. At the later stages of seed maturation, especially for orthodox seeds, AQPs provide roles in rapid water efflux rate, leading to seed desiccation and the accumulation of large amounts of dry matter (Footitt et al., 2019; Hoai et al., 2020). Many AQP genes are actively expressed in diverse seed tissues and during different phases of seed development, according to transcription studies. Transcripts of AtPIP1;2, AtPIP1;3, AtPIP1;4,AtPIP1;5, AtPIP2;1, AtPIP2;2/2;3, AtPIP2;5, AtPIP2;7/AtPIP2;8, AtTIP1;1, AtTIP2;2,AtTIP3;1, AtSIP1;1, AtSIP1;2 and AtSIP2;1 are abundant in one or more seed tissues and at certain seed development stages in Arabidopsis (Hoai et al., 2020).

Many aquaporin genes are strongly expressed in the early periods of seed development, like in the suspensors of Pinus taeda;PsPIP1;1, PsPIP2;1, PsTIP1;1 and PsNIP1;1 in the growing cotyledons and seed coats of 
Table 2. Role of AQPs in the growth of reproductive organs development.

\begin{tabular}{|c|c|c|c|c|c|}
\hline Organs & $\begin{array}{l}\text { Transgenic plants } \\
\text { or mutants }\end{array}$ & Receptors & Phenotypes & AQP Functions & Reference \\
\hline Anthers & $\mathrm{R}:$ NtPIP2 & $\begin{array}{l}\text { Nicotiana taba- } \\
\text { cum }\end{array}$ & $\begin{array}{l}\text { Delayed anther } \\
\text { dehydration } \\
\text { and } \\
\text { dehiscence }\end{array}$ & Water transport & Bots et al., 2005 \\
\hline Pollen & $\begin{array}{l}\text { M: attip1;3 } \\
\text { M: attip5;1 } \\
\text { M: attip1;3/5;1 }\end{array}$ & $\begin{array}{l}\text { Arabidopsis } \\
\text { thaliana }\end{array}$ & $\begin{array}{l}\text { Inhibited pollen } \\
\text { tube elongation }\end{array}$ & $\begin{array}{l}\text { Nitrogen } \\
\text { Transport }\end{array}$ & Soto et al.,2010 \\
\hline \multirow[t]{3}{*}{$\begin{array}{l}\text { Seeds/ } \\
\text { Fruits }\end{array}$} & $\begin{array}{l}\text { O (high levels): } \\
\text { OsPIP } 1 ; 1\end{array}$ & Oryza. sativa & $\begin{array}{l}\text { Decreased } \\
\text { seed yield }\end{array}$ & $\begin{array}{l}\text { A putative water } \\
\text { Transporter }\end{array}$ & Liu et al., 2013 \\
\hline & $\begin{array}{l}\text { O (low or medium } \\
\text { levels): OsPIP } 1 ; 1\end{array}$ & O. sativa & $\begin{array}{l}\text { Increased seed } \\
\text { yield }\end{array}$ & $\begin{array}{l}\text { A putative water } \\
\text { Transporter }\end{array}$ & Liu et al., 2013 \\
\hline & $\mathrm{O}: \mathrm{MdPIP} 1 ; 3$ & $\begin{array}{l}\text { Solanum lyco- } \\
\text { persicum }\end{array}$ & $\begin{array}{l}\text { Promoted ex- } \\
\text { panding } \\
\text { growth of fruits }\end{array}$ & Water transport & Wang et al., 2019 \\
\hline \multirow[t]{3}{*}{ Roots } & $\mathrm{K}:$ AtPIP1 & A. thaliana & Abundant roots & Water transport & Kaldenhoff et al., 2012 \\
\hline & O: PgTIP1 & Panax. ginseng & $\begin{array}{l}\text { Increased pri- } \\
\text { mary root } \\
\text { length }\end{array}$ & Water transport & Lin et al., 2007 \\
\hline & $\mathrm{O}:$ AtPIP2;1 & A. thaliana & $\begin{array}{l}\text { Delayed lateral } \\
\text { root develop- } \\
\text { ment }\end{array}$ & Water transport & Péret et al., 2012 \\
\hline \multirow[t]{3}{*}{$\begin{array}{l}\text { Shoots/ } \\
\text { Stems/ Hypo- } \\
\text { cotyls }\end{array}$} & $\mathrm{O}:$ AtPIP1b & N. tabacum & $\begin{array}{l}\text { Increased } \\
\text { length and } \\
\text { number of } \\
\text { shoot inter- } \\
\text { nodes and stem } \\
\text { diameter }\end{array}$ & Water transport & Aharon et al., 2003 \\
\hline & $\mathrm{R}: B n \mathrm{PIP} 1$ & N. tabacum & $\begin{array}{l}\text { Thicker and } \\
\text { shorter stem }\end{array}$ & Water transport & Yu et al., 2005 \\
\hline & M: tls1 & Zea mays & $\begin{array}{l}\text { Boric acid } \\
\text { transport }\end{array}$ & - & Durbak et al., 2014 \\
\hline Leaves & $\mathrm{O}: T d \mathrm{PIP} 2 ; 1$ & $\begin{array}{l}\text { Triticum tur- } \\
\text { gidum }\end{array}$ & $\begin{array}{l}\text { Enhanced leaf } \\
\text { growth }\end{array}$ & - & Ayadi et al., 2019 \\
\hline
\end{tabular}

pea (Schuurmans et al., 2003);LePIP1;1, LePIP1;2, LePIP1;4, LePIP1;5 and LePIP2;1 in tomato (Shiota et al., 2006); PvPIP1;1, PvPIP2;2 and PvPIP2;3 in seed coats of French bean (Zhou et al., 2007); and OsPIP1;1, OsPIP1;2, OsPIP2;1, OsPIP2;2, OsPIP2;6, OsTIP2;2,OsTIP4;2, and OsNIP1;1 in rice (Hayashi et al., 2015) pointing to the potential roles of these AQPs in seed development.

Lu et al. (2018) reported that GmPIP2;9 is significantly expressed in developing pods and the seed hilum, where assimilation and water transport occur. GmPIP2;9 contains a lot of water channels. Furthermore, overexpression of GmPIP2;9 results in substantial increases in the number of pods, seeds, and seed weight per plant, indicating that GmPIP2;9 may enhance water flow through pod walls from the seed coat and play a role in soybean seed development, seed setting, and seed filling.

Overexpression of OsPIP1;2 in rice significantly increases the number of spikelets per panicle and yield (Table 2).The major mechanism for this may be that OsPIP1;2 favours mesophyll CO2 conductance, further causing enhancements in net $\mathrm{CO} 2$ assimilation rate, photosynthetic capacity and phloem sucrose transport
(Xu et al., 2019). Moreover, overexpression of Panax ginseng PgTIP1 in Arabidopsis promotes seed development (Table 2) (Lin et al., 2007).

Aquaporins may facilitate desiccation during seed development. It may be due to the closing of TIP3;1 aquaporin gene at the maturation stage of seed development. Whereas during seed germination time, phosphorylation of PVTIP3; 1 aquaporin gene facilitates the uptake of water into the protein bodies. It may be due to the opening of TIP3;1 and it is suggested that during seed development time, the TIP3;1 aquaporin gene closed means the water efflux rate is higher, whereasTIP3;1open the water influx rate is higher (Daniels and Yeager, 2005) (Fig. 4).

Role of AQPs during seed maturation and longevity Seed maturation is also aided by AQPs. Up to $90 \%$ of water is lost during the maturity of orthodox seeds. Enormous vacuoles (LVs) transform into protein storage vacuoles (PSVs) at the same time, accumulating large amounts of protein reserves in the PSVs. PSV development is accompanied by a significant rise in TIP3 composition, while TIP1 composition decreases substantially in seeds. The expression of TIP3s and 


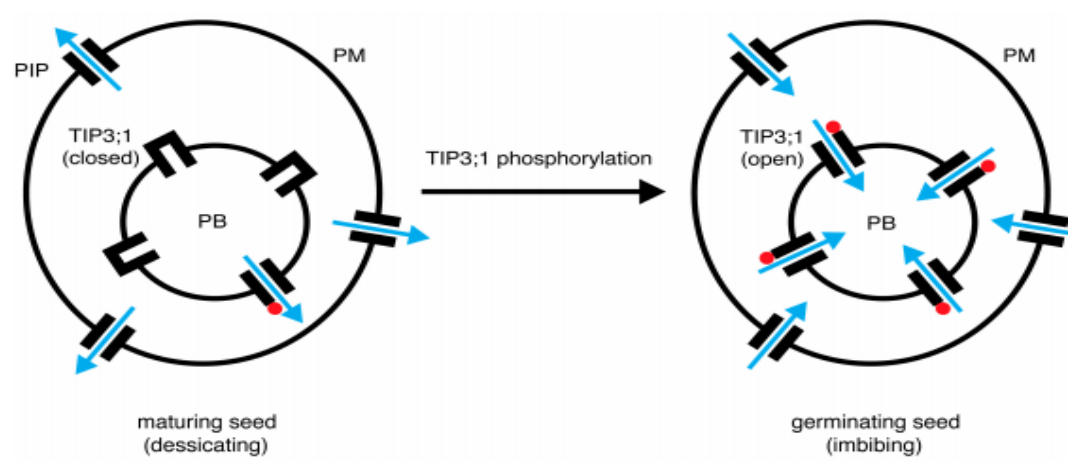

Fig. 4. A model for the role of PVTIP3; 1 aquaporin phosphorylation of seed development in Phaseolus vulgaris (Source: Daniels and Yeager, 2005)

TIP1s genes, on the other hand, is significantly changed (Gattolin et al., 2011; Feeney et al., 2018).

In Arabidopsis, the aquaporin proteins ofAtTIP3;1 and AtTIP3;2 are found to be abundant during the later stage of seed maturation (Willigen et al., 2006). Furthermore, both AtTIP3;1 and AtTIP3;2 gene disruptions lower seed life and increase $\mathrm{H} 2 \mathrm{O} 2$ buildup in seeds. In the presence of $A B A$, the transcription factor $A B A$ insensitive 3 ( $A B \mid 3)$, a key regulator of seed maturation, may bind and activate the promoters of AtTIP3;1 and AtTIP3;2. By transferring water and $\mathrm{H} 2 \mathrm{O} 2$ under the influence of ABA, AtTIP3;1 and AtTIP3;2 operate as positive modulators of seed development and seed lifespan. However, because of their degradation effects on lipids and proteins, reactive oxygen species (ROS) are harmful to seed life.ABI3 play an essential role in seed longevity through the regulation expression of TIP3, late embryogenic abundant (LEA) and heat shock proteins (HSP) (Mao et al., 2015).

Hayashi et al. (2015) discovered that rice OsPIP2;1, OsTIP2;2, and OsTIP3;1 aquaporin proteins are prevalent at the mid-grain filling stage. OsPIP2; 1 is primarily found during rice grain filling in the starchy endosperm, nucellar epidermis, nucellar projection, and dorsal vascular bundles, whereas OsTIP3;1 is mostly found in the aleurone layer and starchy endosperm. Furthermore, following the middle ripening stage of seed development, barley HVTIP3;1 is highly expressed, with its expression peaking during seed desiccation. When coexpressed with HvTIP1;2 in Xenopus oocytes, HvTIP3;1 proteins concentrate in aleurone cells and the outer layers of the seed coat, and they have water permeability (Utsugi et al., 2015).

\section{Role of AQPs in seed dormancy}

According to Footitt et al. (2019), the genetic evidence of AtTIP3;1, AtTIP3;2, and AtTIP4;1 is implicated in seed dormancy regulation. In initial dormancy induction, all three TIPs act as inhibitors. In the induction of secondary dormancy, AtTIP3;1 and AtTIP3;2 have a negative effect.In Arabidopsis, AtTIP3;2 and AtTIP4;1 have inhibitory effects, whereas AtTIP3;1 has promoting effects on secondary dormancy induction. During seasonal dormancy cycling, the expression of the aquaporin genes AtTIP3;1 and AtTIP3;2 changes as well. When seed dormancy levels increased, so did the degree of aquaporin gene expression.

\section{Role of AQPs in seed germination}

AQPs are facilitates water uptake and transport during seed germination (Obroucheva et al., 2013). Many TIP1, TIP2, and PIP genes are substantially expressed up regulated in mRNA or protein levels during seed germination in many crop species, but TIP3s are significantly down regulated. The up regulated genes mainly include AtTIP1;1, AtTIP1;2, AtTIP2;1, AtTIP2;2, AtPIP1;1, AtPIP1;2, AtPIP1;3, AtPIP1;4, AtPIP2;1, AtPIP2;2 and AtPIP2;7 in Arabidopsis (Willigen et al., 2006); OsTIP1;1, OsTIP1;2, OsPIP1;1, OsPIP1;2, OsPIP1;3, OsPIP2;1, OsPIP2;4, OsPIP2;5, OsPIP2;7 and OsPIP2;8 in rice (Li et al., 2018); PsTIP1;1 and PsPIP1;1 in pea (Schuurmans et al., 2003); BnTIP2, BnPIP1 and BnPIP1;4 in oilseed rape (Ge et al., 2014); and VfTIP1;1, VfTIP2;1, VfTIP2;2 and VfPIP2;1 in broad bean (Novikova et al., 2014). In contrast, transcripts of AtTIP3;1 and AtTIP3;2 of Arabidopsis (Willigen et al., 2006), OsTIP3;1 and OsTIP3;2 of rice (Li et al., 2008); and VfTIP3;1 and VfTIP3;2 of broad bean (Novikova et al., 2014) significantly decline during seed germination.

In germinating seeds, some aquaporin TIPs like TIP1s have been shown to function in vacuolar biosynthesis and facilitating water flow into vacuoles, causing the mobilization of reserve substances, the establishment and sustainment of cell turgor pressure and the promotion of embryo cell elongation. Many PIPs such as PIP1s and PIP2s are found to act in water exchange between extracellular and cytoplasmic compartments and are a necessary for water balance maintenance in the cytoplasm (Obroucheva et al., 2010). 
Aquaporins impact vacuolation and $\alpha$-amylase activity, which are necessary for conventional seed germination. Vacuolation happens during seed germination. TIP3s are found mostly in tiny protein storage vacuoles (PSVs), while TIP1s are found mostly in large vacuoles (LVs). TIP3s have a detrimental impact on the development of LVs. By decreasing HvTIP3;1 expression, gibberellins (GAs) stimulate -amylase activity and increase the conversion of PSVs to LVs, whereas abscisic acid (ABA) has the reverse effect during barley seed germination (Lee et al., 2015). AtTIP3;1 and AtTIP4;1 have positive effects, whereas AtTIP3;2 has negative effects on ABA-inhibited seed germination (Footitt et al., 2019). Rice OsPIP1;1 has a beneficial role in seed germination by inducing $\alpha$-amylase activity, and OsPIP1;3 plays a positive role during seed germination (Liu et al., 2013). TIP1 abundance is favourably linked with LV development, while TIP3 abundance is adversely correlated. TIP3s, on the other hand, have been reported to favour optimum water absorption in Vicia faba seedlings during the early stages of germination (Bere et al., 2017) (Fig. 5).

AQPs also play a role in recalcitrant seed germination. According to Obroucheva et al. (2012), AhTIP2, AhTIP3;1, AhPIP1 and AhPIP2 are prevalent during horse chestnut seed germination. Active vacuoles develop in the embryonic axis cells of hypocotyls and radicles after seed shedding, followed by vacuolation during seed germination. Furthermore, the activation of vacuolar acid invertase causes cell vacuolation.

\section{Role of AQPs under abiotic stress \\ Drought stress}

AQPs are altered the expression patterns and dynamic changes in subcellular localization have been observed in plants in response to drought stress (Maurel et al., 2008). Long-term root exposure to drought-stressed soil causes aquaporin activity to be inhibited, preventing reverse water transfer from the root cells to the dry soil (Maurel et al., 2015; Afzal et al., 2016). Further- more, early drought circumstances induced aquaporin activity and expression in order to maximise the uptake of available soil water and combat water scarcity (Shekoofa and Sinclair, 2018). Under drought stress, the expression profile of Arabidopsis whole 35 aquaporin-encoding genes was examined, and it was discovered that most PIPs and TIPs were up-regulated, whilst transcription of NIPs was down-regulated (Alexandersson et al., 2005). Some aquaporins, such as AtPIP2;6 and AtSIP1;1, were shown to be constitutive and unaffected by water deficiency. Drought, in general, is linked to heat stress and causes more severe damage to agricultural plants than either condition alone (Rizhsky et al., 2002). In Arabidopsis, the combined effect of heat and drought up-regulated many Heat Shock proteins (HSPs), protein kinases, stressresponsive transcription factors, and MIPs. (Rizhsky et al., 2004). PIPs and TIPs are the major class of aquaporins whose expression is up-regulated during drought in roots, leaves, flowers, and seeds, according to comparative transcriptome studies from various plants (Deshmukh et al., 2016).

In maize, arbuscular mycorrhizal symbiosis differently controlled the expression of various PIPs and NIPs in response to short-term and long-term drought (Barzana et al., 2014). Under water stress, PIP activity in roots influences hydraulic characteristics, including root hydraulic conductance (Lpr) and root sap exudation rate $(\mathrm{Sr})$. Aquaporins produced in xylem parenchyma cells are functionally engaged in restoring xylem hydraulic conductivity during the recovery phase from water stress (Secchi et al., 2017).

\section{Cold stress}

Root hydraulic conductance (Lpr) and sap flow in the roots is reduced when plants are exposed to cold temperatures or chilling stress $\left(4-8{ }^{\circ} \mathrm{C}\right)$. Plants that are resistant to cold stress may recover, but sensitive plants will not be able to do so and may die as a result. In Arabidopsis, rice, and maize, transcriptome analysis of root and leaf revealed a substantial decrease in expression

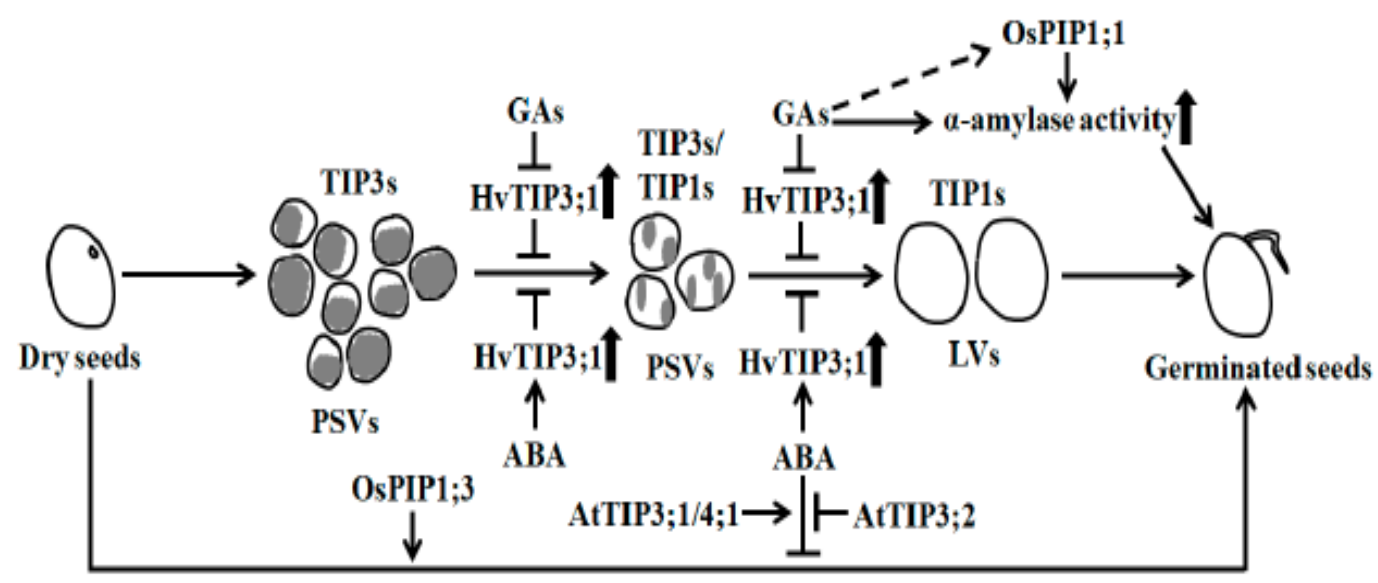

Fig. 5. Role of $A Q P s$ during orthodox seed germination (Source: Wang et al., 2020). 
of most aquaporin genes in response to cold stress (Jang et al., 2004; Sakurai et al., 2005; Yu et al., 2006). The expression of root-specific aquaporin PIP genes, on the other hand, has been demonstrated to be upregulated throughout the healing phase. Relative expression of OsPIP2;5 was superior in the long-term low temperature treated plants in rice (Ahamed et al., 2012).In cold-tolerant banana species, the early cold response induced increased expression of certain PIPs and TIPs, such as MaPIP1;1, MaPIP1;2, MaPIP2;4, MaPIP2;6, MaTIP1;3, which help maintain leaf water potential and adapt to low temperatures (He et al., 2018).

\section{Salinity stress}

The salinity stress results in impaired osmotic potential in plant cells; affects growth and development, plant metabolism and yield. Liang et al.2018 found that the early reaction of plants to salt stress is a restriction of water absorption capacity through roots, resulting in a decrease in root hydraulic conductance (Lpr). Salt treatment resulted in a rapid and significant decrease in Lpr and transcription of PIP and TIP generating genes in Arabidopsis roots. (Boursiac et al., 2005). Barley has also been found to have downregulated aquaporin gene expression in response to salt stress (Katsuhara et al., 2002).In the presence of $100 \mathrm{mM} \mathrm{NaCl}$, transient ABA-dependent induction of ZmPIP1;2 and ZmPIP2;4 and $\mathrm{ABA}$-independent upregulation of $\mathrm{ZmPIP1;1}$ and ZmPIP1;5 were seen in maize (Zhu et al., 2005). The expression of none of the TIPs, on the other hand, was altered. Regardless of the presence of ABA, exogenous administration of $200 \mathrm{mM} \mathrm{NaCl}$ after $24 \mathrm{~h}$ resulted in the inhibition of most of the PIPs and TIPs aquaporins genes. TIPs play a key role in the transport of cytoplasmic ROS molecules, urea, ammonium, and nitrate ions, as well as water, into the vacuolar region (Afzal et al., 2016). Salt stress in Arabidopsis roots may cause partial subcellular translocation of TIPs in the intravacuolar region (Boursiac et al., 2005). In wheat, TaNIP aquaporin was shown to be highly up regulated in salinity treatment (Gao et al., 1999). Most aquaporins in citrus were up regulated by salt treatment in roots and either up regulated or down regulated in leaves, according to Martins et al. (2015). Water inflow into the leaves may be aided by the aquaporins produced in leaves by salt treatment (Fig. 6).

\section{Conclusion}

AQPs are involved in plant growth and development functions, such as water and nutrients transport, nitrogen, carbon, and micronutrient acquisition. AQPs expression modulates the water, mineral nutrient uptake, utilization, improves water and nutrient use efficiency in

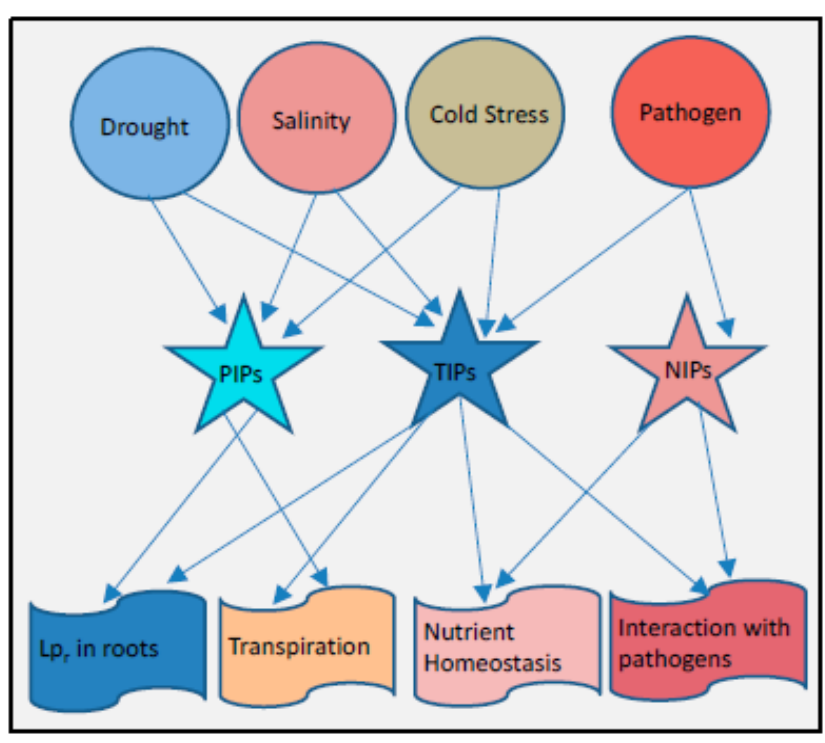

Fig. 6. Role of $A Q P$ s under abiotic stress (Source: Liang et al., 2018).

plants, and increases tolerance to abiotic stress. Different aquaporins found in the seed system have individual functions that are more time and tissue specific, ultimately helping in the seed imbibition process to complete seed germination. Seed specific TIP3s helps in maintain seed longevity under the expressional control of $\mathrm{ABI} 3$ during seed maturation together with late embryogenic abundant (LEA) and heat shock proteins (HSP). The role of aquaporins during biotic and abiotic stress; and the relevance of altered aquaporin expression for biotechnological improvement of plant tolerance must be explored. The molecular mechanism of aquaporin in seeds needs to be studied. The detailed mechanisms for most of the AQPs are still not clear. Furthermore, it is yet unknown if and how other plant growth hormones such as cytokinin, brassinosteroid, and salicylic acid impact the expression and activity of AQPs, therefore modulating plant growth and development. AQPs are currently regarded as a promising candidate gene for engineering to increase stress tolerance and agricultural production. As a result, greater effort should be put into better understanding the complex regulation mechanisms of AQPs in order to maximise the effective usage of water and nutrient transport for healthy plant growth and development, especially under stress conditions.

\section{Conflict of interest}

The authors declare that they have no conflict of interest.

\section{REFERENCES}

1. Afzal, Z., Howton, T.C., Sun, Y. \& Mukhtar, M.S. (2016). The roles of aquaporins in plant stress responses. Journal of Developmental Biology, 4(1), 9. https:// doi.org/ 
10.3390/jdb4010009.

2. Ahamed, A., Murai-Hatano, M., Ishikawa-Sakurai, J., Hayashi, H., Kawamura, Y. \& Uemura, M. (2012). Cold stress-induced acclimation in rice is mediated by rootspecific aquaporins. Plant and Cell Physiology, 53(8), 1445-1456. https://doi.org/ 10.1093/pcp/pcs089.

3. Aharon, R., Shahak, Y., Wininger, S., Bendov, R., Kapulnik, Y. \& Galili, G. (2003). Overexpression of a plasma membrane aquaporin in transgenic tobacco improves plant vigor under favorable growth conditions but not under drought or salt stress. The Plant Cell, 15(2), 439-447. https://doi.org/10.1105/tpc.009225.

4. Alexandersson, E., Fraysse, L., Sjövall-Larsen, S., Gustavsson, S., Fellert, M., Karlsson, M., Johanson, U. \& Kjellbom, P. (2005). Whole gene family expression and drought stress regulation of aquaporins. Plant Molecular Biology, 59(3), 469-484. DOI 10.1007/s11103-005-03 52-1.

5. Ayadi, M., Brini, F. \& Masmoudi, K. (2019). Overexpression of a wheat aquaporin gene, TdPIP2; 1 , enhances salt and drought tolerance in transgenic durum wheat $\mathrm{cv}$. maali. International Journal of Molecular Sciences, 20(10), 2389. https://doi.org/10.3390/ijms20102389.

6. Barzana, G., Aroca, R., Bienert, G.P., Chaumont, F. \& Ruiz-Lozano, J.M. (2014). New insights into the regulation of aquaporins by the arbuscular mycorrhizal symbiosis in maize plants under drought stress and possible implications for plant performance. Molecular Plant-Microbe Interactions, 27(4), 349-363. https://doi.org/ 10.1094/MPMI-0913-0268-R.

7. Bere, E., Lahbib, K., Merceron, B., Fleurat-Lessard, P. \& Boughanmi, N.G. (2017). a-TIP aquaporin distribution and size tonoplast variation in storage cells of Vicia faba cotyledons at seed maturation and germination stages. Journal of Plant Physiology, 216, 145-151. https:// doi.org/10.1016/j.jplph.2017.04.019.

8. Bots, M., Vergeldt, F., Wolters-Arts, M., Weterings, K., van As, H. \& Mariani, C., (2005). Aquaporins of the PIP2 class are required for efficient anther dehiscence in tobacco. Plant Physiology, 137(3), 1049-1056. https:// doi.org/10.1104/pp.104.056408

9. Boursiac, Y., Chen, S., Luu, D.T., Sorieul, M., van den Dries, N. \& Maurel, C. (2005). Early effects of salinity on water transport in Arabidopsis roots. Molecular and cellular features of aquaporin expression. Plant Physiology, 139(2), 790-805. https://doi.org/10.1104/pp.105.0 650 29.

10. Chen, W., Yin, X., Wang, L., Tian, J., Yang, R., Liu, D., Yu, Z., Ma, N. \& Gao, J. (2013). Involvement of rose aquaporin RhPIP1; 1 in ethylene-regulated petal expansion through interaction with RhPIP2; 1. Plant Molecular Biology, 83(3), 219-233. DOI 10.1007/s11103-013-0084-6.

11. Daniels, M.J. \& Yeager, M. (2005). Phosphorylation of aquaporin PVTIP3; 1 defined by mass spectrometry and molecular modeling. Biochemistry, 44(44), 14443-14454. https://doi.org/10.1021/bi050565d

12. Deshmukh, R.K., Sonah, H. \& Bélanger, R.R. (2016). Plant Aquaporins: genome-wide identification, transcriptomics, proteomics, and advanced analytical tools. Frontiers in Plant Science, 7, 1896. https:// doi.org/10.3389/fpls.2016.01896.

13. Dixit, R., Rizzo, C., Nasrallah, M. \& Nasrallah, J. (2001).
The Brassica MIP-MOD gene encodes a functional water channel that is expressed in the stigma epidermis. Plant Molecular Biology, 45(1), 51-62.

14. Dubois, M., Broeck, L.V.D. \& Inze, D. (2018). The pivotal role of ethylene in plantgrowth. Trends Plant Science, 23, 311-323. https://doi.org/10.1016/j.tplants.2018.01.003.

15. Durbak, A.R., Phillips, K.A., Pike, S., O’Neill, M.A., Mares, J., Gallavotti, A., Malcomber, S.T., Gassmann, W. \& McSteen, P. (2014). Transport of boron by the tasselless 1 aquaporin is critical for vegetative and reproductive development in maize. The Plant Cell, 26(7), 2978-2995. https://doi.org/10.1105/tpc.114.125898.

16. Feeney, M., Kittelmann, M., Menassa, R., Hawes, C. \& Frigerio, L. (2018). Protein storage vacuoles originate from remodeled preexisting vacuoles in Arabidopsis thaliana. Plant Physiology, 177(1), 241-254. https:// doi.org/10.1104/pp.18.00010.

17. Footitt, S., Clewes, R., Feeney, M., Finch $\square$ Savage, W.E. \& Frigerio, L. (2019). Aquaporins influence seed dormancy and germination in response to stress. Plant, Cell \& Environment, 42(8), 2325-2339. https://doi.org/10.1111/ pce.13561.

18. Gao, Y.P., Young, L., Bonham-Smith, P. \& Gusta, L.V. (1999). Characterization and expression of plasma and tonoplast membrane aquaporins in primed seed of Brassica napus during germination under stress conditions. Plant Molecular Biology, 40(4), 635-644.

19. Gattolin, S., Sorieul, M. \& Frigerio, L. (2011). Mapping of tonoplast intrinsic proteins in maturing and germinating Arabidopsis seeds reveals dual localization of embryonic TIPs to the tonoplast and plasma membrane. Molecular Plant, 4(1), 180-189. https://doi.org/10.1093/mp/ssq051.

20. Ge, F.W., Tao, P., Zhang, Y. \& Wang, J.B. (2014). Characterization of AQP gene expressions in Brassica napus during seed germination and in response to abiotic stresses. Biologia Plantarum, 58(2), 274-282.

21. Hayashi, H., Ishikawa-Sakurai, J., Murai-Hatano, M., Ahamed, A. \& Uemura, M. (2015). Aquaporins in developing rice grains. Bioscience, Biotechnology, and Biochemistry, 79(9), 1422-1429. https://doi.org/10.1080/09 168451.2015.1032882.

22. He, W.D., Gao, J., Dou, T.X., Shao, X.H., Bi, F.C., Sheng, O., Deng, G.M., Li, C.Y., Hu, C.H., Liu, J.H. \& Zhang, S. (2018). Early cold-induced peroxidases and aquaporins are associated with high cold tolerance in Dajiao (Musa spp.'Dajiao'). Frontiers in Plant Science, 9, 282. https:// doi.org/10.3389/fpls. 2018.00282.

23. Hoai, P.T., Tyerman, S.D., Schnell, N., Tucker, M., McGaughey, S.A., Qiu, J., Groszmann, M. \& Byrt, C.S (2020). Deciphering aquaporin regulation and roles in seed biology. Journal of Experimental Botany, 71(6), 1763 -1773. https://doi.org/ 10.1093/jxb/erz555.

24. Jang, J.Y., Kim, D.G., Kim, Y.O., Kim, J.S. \& Kang, H. (2004). An expression analysis of a gene family encoding plasma membrane aquaporins in response to abiotic stresses in Arabidopsis thaliana. Plant Molecular Biology, 54(5), 713-725.

25. Johansson, I., Karlsson, M., Shukla, V.K., Chrispeels, M.J., Larsson, C. \& Kjellbom, P. (1998). Water transport activity of the plasma membrane aquaporin PM28A is regulated by phosphorylation. The Plant Cell, 10(3), 451459. https://doi.org/10.1105/tpc.10.3.451. 
26. Kaldenhoff, R. (2012). Mechanisms underlying $\mathrm{CO}_{2}$ diffusion in leaves. Current Opinion in Plant Biology, 15(3), 276-281. https://doi.org/10.1016/j.pbi.2012.01.011.

27. Katsuhara, M., Akiyama, Y., Koshio, K., Shibasaka, M. \& Kasamo, K. (2002). Functional analysis of water channels in barley roots. Plant and Cell Physiology, 43(8), 885-893. https://doi.org/10.1093/pcp/pcf102.

28. Knepper, M.A. \& Inoue, T. (1997). Regulation of aquaporin-2 water channel trafficking by vasopressin. Current Opinion in Cell Biology, 9(4), 560-564. https:// doi.org/10.1016/S0955-0674(97)80034-8.

29. Lee, S.E., Yim, H.K., Lim, M.N., Yoon, I.S., Kim, J.H. \& Hwang, Y.S. (2015). Abscisic acid prevents the coalescence of protein storage vacuoles by upregulating expression of a tonoplast intrinsic protein gene in barley aleurone. Journal of Experimental botany, 66(5), 1191-1203. https://doi.org/10.1093/jxb/eru467.

30. Li, W., Qiang, X.J., Han, X.R., Jiang, L.L., Zhang, S.H., Han, J., He, R. \& Cheng, X.G. (2018). Ectopic expression of a Thellungiella salsuginea aquaporin gene, TsPIP1; 1 , increased the salt tolerance of rice. International Journal of Molecular Sciences, 19(8), 2229. https://doi.org/10.33 90/ijms 19082229 .

31. Liang, W., Ma, X., Wan, P. \& Liu, L. (2018). Plant salttolerance mechanism: A review. Biochemical and Biophysical Research Communications, 495(1), 286-291. https://doi.org/10.1016/j.bbrc.2017.11.043.

32. Lin, W., Peng, Y., Li, G., Arora, R., Tang, Z., Su, W. \& Cai, $W$. (2007). Isolation and functional characterization of PgTIP1, a hormone-autotrophic cells-specific tonoplast aquaporin in ginseng. Journal of Experimental Botany, 58 (5), 947-956. https://doi.org/10.1093/jxb/erl255.

33. Liu, C., Fukumoto, T., Matsumoto, T., Gena, P., Frascaria, D., Kaneko, T., Katsuhara, M., Zhong, S., Sun, X., Zhu, Y. \& Iwasaki, I. (2013). Aquaporin OsPIP1; 1 promotes rice salt resistance and seed germination. Plant Physiology and Biochemistry, 63, 151-158. https://doi.org/10.1016/ j.plaphy.2012.11.018.

34. Liu, S., Fukumoto, T., Gena, P., Feng, P., Sun, Q., Li, Q., Matsumoto, T., Kaneko, T., Zhang, H., Zhang, Y. \& Zhong, S. (2020). Ectopic expression of a rice plasma membrane intrinsic protein (OsPIP1; 3) promotes plant growth and water uptake. The Plant Journal, 102(4), 779796. https://doi.org/10.1111/tpj.14662.

35. Lu, L., Dong, C., Liu, R., Zhou, B., Wang, C. \& Shou, H. (2018). Roles of soybean plasma membrane intrinsic protein GmPIP2; 9 in drought tolerance and seed development. Frontiers in Plant Science, 9, 530. https:// doi.org/10.3389/fpls. 2018.00530.

36. Ma, N., Xue, J., Li, Y., Liu, X., Dai, F., Jia, W., Luo, Y. \& Gao, J. (2008). Rh-PIP2; 1, a rose aquaporin gene, is involved in ethylene-regulated petal expansion. Plant Physiology, 148(2), 894-907. https://doi.org/10.1104/pp.10 8.120154

37. Mao, Z. \& Sun, W. (2015). Arabidopsis seed-specific vacuolar aquaporins are involved in maintaining seed longevity under the control of ABSCISIC ACID INSENSITIVE 3. Journal of Experimental Botany, 66(15), 4781-4794. https://doi.org/ 10.1093/jxb/erv244.

38. Martins, C.D.P.S., Pedrosa, A.M., Du, D., Goncalves, L.P., Yu, Q., Gmitter Jr, F.G. \& Costa, M.G.C. (2015).
Genome-wide characterization and expression analysis of major intrinsic proteins during abiotic and biotic stresses in sweet orange (Citrus sinensis L. Osb.). PLOS one, 10(9), 138-146. https://doi.org/10.1371/journal.pon e.0138786

39. Maurel, C., Boursiac, Y., Luu, D.T., Santoni, V., Shahzad, Z. \& Verdoucq, L. (2015). Aquaporins in plants. Physiological Reviews, 95(4), 1321-1358. https:// doi.org/ 10.1152/physrev.00008.2015.

40. Maurel, C., Verdoucq, L., Luu, D.T. \& Santoni, V. (2008). Plant aquaporins: membrane channels with multiple integrated functions. Annual Review of Plant Biology, 59, 595-624.

41. Novikova, G.V., Tournaire-Roux, C., Sinkevich, I.A., Lityagina, S.V., Maurel, C. \& Obroucheva, N. (2014). Vacuolar biogenesis and aquaporin expression at early germination of broad bean seeds. Plant Physiology and Biochemistry, 82, 123-132. https://doi.org/10.1016/ j.plaphy.2014.05.014.

42. Obroucheva, N.V. \& Sin'Kevich, I.A. (2010). Aquaporins and cell growth. Russian Journal of Plant Physiology, 57 (2), 153-165.

43. Obroucheva, N.V. (2013). Aquaporins in seeds. Seed Science Research, 23(4), 213-216.

44. Obroucheva, N.V., Lityagina, S.V., Novikova, G.V. \& Sin'kevich, I.A. (2012). Vacuolar status and water relations in embryonic axes of recalcitrant Aesculus hippocastanum seeds during stratification and early germination. AoB Plants, 23(3), 312-319. https://doi.org/10.1093/ aobpla/pls008.

45. Péret, B., Li, G., Zhao, J., Band, L.R., Voß, U., Postaire, O., Luu, D.T., Da Ines, O., Casimiro, I., Lucas, M. \& Wells, D.M. (2012). Auxin regulates aquaporin function to facilitate lateral root emergence. Nature Cell Biology, 14 (10), 991-998.

46. Rizhsky, L., Liang, H. \& Mittler, R. (2002). The combined effect of drought stress and heat shock on gene expression in tobacco. Plant Physiology, 130(3), 1143-1151. https://doi.org/10.1104/pp.006858.

47. Rizhsky, L., Liang, H., Shuman, J., Shulaev, V., Davletova, S. \& Mittler, R. (2004). When defense pathways collide. The response of Arabidopsis to a combination of drought and heat stress. Plant Physiology, 134(4), 16831696. https://doi.org/ 10.1104/pp.103.033431.

48. Sakurai, J., Ishikawa, F., Yamaguchi, T., Uemura, M. \& Maeshima, M. (2005). Identification of 33 rice aquaporin genes and analysis of their expression and function. Plant and Cell Physiology, 46(9), 1568-1577. https:// doi.org/ 10.1093/ pcp/pci172.

49. Sato, R. \& Maeshima, M. (2019). The ER-localized aquaporin SIP2; 1 is involved in pollen germination and pollen tube elongation in Arabidopsis thaliana. Plant Molecular Biology, 100(3), 335-349.

50. Schuurmans, J.A., van Dongen, J.T., Rutjens, B.P., Boonman, A., Pieterse, C.M. \& Borstlap, A.C. (2003) Members of the aquaporin family in the developing pea seed coat include representatives of the PIP, TIP, and NIP subfamilies. Plant Molecular Biology, 53(5), 655-667.

51. Secchi, F., Pagliarani, C. \& Zwieniecki, M.A. (2017). The functional role of xylem parenchyma cells and aquaporins during recovery from severe water stress. Plant, 
Cell \& Environment, 40(6), 858-871. https://doi.org/10.1 111/pce.12831.

52. Shapiguzov, A.Y. (2004). Aquaporins: structure, systematics, and regulatory features. Russian Journal of Plant Physiology, 51(1), 127-137.

53. Shekoofa, A. \& Sinclair, T.R. (2018). Aquaporin activity to improve crop drought tolerance. Cells, 7(9), 123. https:// doi.org/10.3390/cells7090123.

54. Shiota, H., Sudoh, T. \& Tanaka, I. (2006). Expression analysis of genes encoding plasma membrane aquaporins during seed and fruit development in tomato. Plant Science, 171(2), 277-285. https://doi.org/10.1016/j.plant sci.2006.03.021.

55. Soto, G., Alleva, K., Mazzella, M.A., Amodeo, G. \& Muschietti, J.P. (2008). AtTIP1; 3 and AtTIP5; 1, the only highly expressed Arabidopsis pollen-specific aquaporins, transport water and urea. FEBS letters, 582(29), 40774082. https://doi.org/ 10.1016/j.febslet.2008.11.002.

56. Soto, G., Fox, R., Ayub, N., Alleva, K., Guaimas, F., Erijman, E.J., Mazzella, A., Amodeo, G. \& Muschietti, J. (2010). TIP5; 1 is an aquaporin specifically targeted to pollen mitochondria and is probably involved in nitrogen remobilization in Arabidopsis thaliana. The Plant Journal, 64(6), 1038-1047. https://doi.org/ 10.1111/j.1365313X.2010.04395.x.

57. Utsugi, S., Shibasaka, M., Maekawa, M. \& Katsuhara, M. (2015). Control of the water transport activity of barley HvTIP3; 1 specifically expressed in seeds. Plant and Cell Physiology, 56(9), 1831-1840. https://doi.org/10.1093/pcp/ pcr104.

58. Willigen Vander, C., Postaire, O., Tournaire-Roux, C., Boursiac, Y. \& Maurel, C. (2006). Expression and inhibition of aquaporins in germinating Arabidopsis seeds. Plant and Cell Physiology, 47(9), 1241-1250. https://doi.org/ 10.1093/pcp/pcj094.

59. Wang, X., Gao, F., Bing, J., Sun, W., Feng, X., Ma, X., Zhou, Y. \& Zhang, G. (2019). Overexpression of the Jojoba aquaporin gene, ScPIP1, enhances drought and salt tolerance in transgenic Arabidopsis. International Journal of Molecular Sciences, 20(1), 153. https://doi.org/10.3390/ ijms20040837.
60. Wang, Y., Zhao, Z., Liu, F., Sun, L. \& Hao, F. (2020). Versatile Roles of Aquaporins in Plant Growth and Development. International Journal of Molecular Sciences, 21(24), 9485. https://doi.org/10.3390/ijms21249485.

61. Wudick, M.M., Luu, D.T., Tournaire-Roux, C., Sakamoto, W. \& Maurel, C. (2014). Vegetative and sperm cellspecific aquaporins of Arabidopsis highlight the vacuolar equipment of pollen and contribute to plant reproduction. Plant Physiology, 164(4), 1697-1706. https:// doi.org/10.1104/pp.113.228700.

62. Xu, F., Wang, K., Yuan, W., Xu, W., Liu, S., Kronzucker, H.J., Chen, G., Miao, R., Zhang, M., Ding, M. \& Xiao, L. (2019). Overexpression of rice aquaporin OsPIP1; $2 \mathrm{im}$ proves yield by enhancing mesophyll $\mathrm{CO} 2$ conductance and phloem sucrose transport. Journal of Experimental Botany, 70(2), 671-681. https://doi.org/ 10.1093/jxb/ ery386.

63. Xue, J., Yang, F. \& Gao, J. (2009). Isolation of Rh-TIP1; 1, an aquaporin gene and its expression in rose flowers in response to ethylene and water deficit. Postharvest Biology and Technology, 51(3), 407-413. https:// doi.org/10.1016/j.postharvbio. 2008.08.011.

64. Yu, Q., Hu, Y., Li, J., Wu, Q. \& Lin, Z. (2005). Sense and antisense expression of plasma membrane aquaporin BnPIP1 from Brassica napus in tobacco and its effects on plant drought resistance. Plant Science, 169(4), 647-656. https://doi.org/10.1016/j.plantsci.2005.04.013.

65. Yu, X., Peng, Y.H., Zhang, M.H., Shao, Y.J., Su, W.A. \& Tang, Z.C. (2006). Water relations and an expression analysis of plasma membrane intrinsic proteins in sensitive and tolerant rice during chilling and recovery. Cell Research, 16(6), 599-608

66. Zhou, Y., Setz, N., Niemietz, C., Qu, H., Offler, C.E., Tyerman, S.D. \& Patrick, J.W. (2007). Aquaporins and unloading of phloem-imported water in coats of developing bean seeds. Plant, Cell \& Environment, 30(12), 1566-1577. https://doi.org/ 10.1111/ j.1365-3040.2007.01732.x.

67. Zhu, C., Schraut, D., Hartung, W. \& Schäffner, A.R. (2005). Differential responses of maize MIP genes to salt stress and ABA. Journal of Experimental Botany, 56(421), 2971-2981. https://doi.org/10.1093/jxb/eri294. 